\title{
2-D DOA Estimation for Acoustic Vector Sensor Array Based on Greedy Block Coordinate Descent Algorithm
}

\author{
Jie $\mathrm{Hu}^{1, \mathrm{a}}$,Wei $\mathrm{Li}^{2, \mathrm{~b}}$ and Shaojun $\mathrm{zuo}^{3, \mathrm{c}}$ \\ ${ }^{1}$ Department of Electronic and Information Engineering, Harbin Institute of Technology Shenzhen \\ Graduate School, Shenzhen, China \\ ${ }^{2}$ Department of Electronic and Information Engineering, Harbin Institute of Technology Shenzhen \\ Graduate School, Shenzhen, China \\ ${ }^{3}$ Department of Electronic and Information Engineering, Harbin Institute of Technology Shenzhen \\ Graduate School, Shenzhen, China \\ ahujie1216@yeah.net, ${ }^{b}$ li.wei@hitsz.edu.cn, c nuptzuo@163.com
}

Keywords: two-dimensional direction of arrival (DOA) estimation, acoustic vector sensor (AVS), greedy block coordinate descent(GBCD).

\begin{abstract}
This paper presents an approach for the estimation of two-directional (2D)direction-of-arrival (DOA) using Acoustic Vector Sensor array based on greedy block coordinate descent(GBCD) algorithm, which can achieve faster convergence rate and better estimation accuracy. Moreover, a weighted form of block selection rule is proposed with the MUSIC prior. The identifiability of the presented approach is studied using computer simulations. It is demonstrated that the 2D DOAs of AVS can be realized using the approach, which has a superior resolution.
\end{abstract}

\section{Introduction}

Two-dimensional (2D) direction of arrival (DOA) estimation with array sensors is essential for source localization in audio surveillance, auditory scene analysis, hearing aids etc. The most well-known classical DOA estimation methods include Capon's method [1], MUSIC [2], ESPRIT [3], etc. The four-element Acoustic Vector Sensor (AVS) first presented in [4] for DOA estimation is an acoustic sensor that is capable of measuring acoustic pressure gradient as well as pressure as in a standard microphone. This combination makes it possible for the sensor to measure the complete sound field. Recently, along with the development of AVS technique, the Direction of Arrival (DOA) estimation based on AVS has been researched widely and deeply [5-8].

By introducing Compressed Sensing (CS) [9] into DOA estimation field, we can obtain better performance than traditional angle estimation methods using the prior sparse information of target scene. In 2011, Fu applied CS to the DOA estimation of AVS [10], the echo signal received by an uniform linear array (ULA) is used for recovering target angle through OMP algorithm. But limited by the inherent error probability, OMP is not stable under noisy environment. Liang proposed an acoustic vector sensor array space time filtering approach for azimuth estimation with $l_{1}$-norm optimization [11] and obtains superior resolution and accuracy to the conventional methods. However, the huge computational complexity of $l_{1}$-norm optimization restricts potential application.

Aiming at these problems, 2D DOA estimation for AVS based on GBCD algorithm is studied in this paper. In section 2, the acoustic vector sensor array signal model is introduced. Simulations are given in section 3 and summary in section 4 .

\section{Signal Model}

Acoustic Vector Sensor Array Signal Model. An acoustic vector sensor consists of an acoustic pressure channel and three orthogonally oriented acoustic velocity channels, respectively receive 
the acoustic pressure $\mathrm{P}$ and three components $\left(v_{x}, v_{y}, v_{z}\right)$ of the acoustic particle velocity on coordinate axes $\mathrm{x}, \mathrm{y}, \mathrm{x}$. We assume there is a linear array with $\mathrm{N}$ vector sensors. The output of each AVS is a 4-dimensional vector which contains all the 4 components. We assume $\mathrm{K}$ far-field sources are in the surveillance region, impinging on this linear array from directions, where $\phi_{k}$ and $\theta_{k}$ represent the azimuth and the elevation angles of the kth signal respectively. We assume $-\frac{\pi}{2} \leq \theta \leq \frac{\pi}{2}$ and $-\pi \leq \phi \leq \pi$. The measurement received at the array at time $\mathrm{t}$ can be modeled as [12]

$$
y(t)=A x(t)+e(t)
$$

where $y(t)$ and $e(t)$ are $4 N \times 1$ complex vectors, respectively, and $x(t)$ is the $K \times 1$ source vector.

The $4 N \times K$ array manifold $A$ can be expressed as

$$
A=\left[a_{1}, \ldots . ., a_{k}\right]
$$

Where $a_{k}=d_{k} \otimes p_{k}$, with

$$
\begin{aligned}
& d_{k}=\left[1, e^{j 2 \pi \sin \theta k}, \ldots, e^{j(N-1) \pi \sin \theta k}\right]^{T} \\
& p_{k}=\left[1, \cos \phi_{k} \sin \theta_{k}, \sin \phi_{k} \sin \theta_{k}, \sin \theta_{k}\right]^{T}
\end{aligned}
$$

Here, $a_{k}$ is the $4 N \times 1$ steering vector of the array associated with a signal coming from the direction $\left(\phi_{k}, \theta_{k}\right) . d_{k}$ denotes the phase delay of the kth signal at the $\mathrm{N}$ sensors with respect to the origin, $p_{k}$ is the steering vector of a single vector sensor located at the origin.

Joint Sparse Signal Model of Acoustic Vector Sensor Array. Supposing we process L snapshots at a time, the echo vector, source vector and noise vector received at the lth snapshot can be represented by $y^{(l)}, s^{(l)}$ and $e^{(l)}$ respectively, which independent from each other between different snapshots $l=1, \ldots, L$, we can get

$$
\begin{aligned}
& Y:=\left[y^{(1)} \ldots y^{(L)}\right]=\Phi S+E \\
& \Phi=\left[d_{1} \otimes p_{11}, \ldots, d_{p} \otimes p_{p 1}, \ldots, d_{1} \otimes p_{1 M}, \ldots, d_{p} \otimes p_{p M}\right] \in R^{4 N \times P M}
\end{aligned}
$$

where $S=\left[s^{(1)} \ldots . s^{(L)}\right]$ is the source matrix, $E=\left[e^{(1)} \ldots e^{(L)}\right]$ is the noise matrix.

Compared with the 1-D overcomplete dictionary, 2-D overcomplete dictionary is designed by expanding the overcomplete basis in 1-D way. Basing on the spatial sparsity, in each snapshot, $s^{(l)}$ has only few nonzero elements, and the targets haven't moved cross resolution units during $\mathrm{L}$ snapshots, that means for any $s^{(i)}$ and $s^{(j)}(i \neq j)$, the nonzero elements are at the same position, so there are only few rows in $S$ which are nonzero, and (3) is called the joint row sparse signal model of acoustic vector sensor array.

Singular Value Decomposition. In order to reduce the dimension of (3), we apply Singular Value Decomposition to the echo matrix

$$
Y=U \Lambda V^{H}=\left[U_{s} U_{N}\right] \Lambda V^{H}
$$

where $U_{s}$ is the signal subspace matrix and $U_{N}$ is the noise subspace matrix. $U_{s}$ consists of the eigenvectors correspond to the former $\mathrm{K}$ large eigenvalues and $U_{N}$ consists of the eigenvectors correspond to the rest L-K small eigenvalues. Let $D_{k}=\left[\Lambda_{K \times K}^{-1} 0_{K \times(L-K)}^{H}\right]^{H}$, where $\Lambda_{K \times K}$ represents the diagonal matrix constructed by the former $\mathrm{K}$ large eigenvalues, thus the signal subspace matrix can be represented as $U_{s}=Y V D_{k}$. Let $Y_{s}=U_{s}, S_{s}=S V D_{k}, E_{s}=E V D_{k}$, substituted into (3), there is the dimensional reduction representation

$$
Y_{s}=\Phi S_{s}+E_{s}
$$

Through comparing (5) with (3), we know that the dimension of echo signal is reduced from $3 P \times L$ to $3 P \times K$. In practice, $K<<L$, so the calculation of (5) is much faster. This fact motivates 
the following $l_{2,1}$-norm minimization formulation for jointly sparse signal recovery [13]:

$$
\min \frac{1}{2}\left\|Y_{s}-\Phi S_{s}\right\|_{F}^{2}+\lambda\left\|S_{s}\right\|_{2,1}
$$

Where $\|\bullet\|_{F}$ denotes the matrix Frobenius norm, $\left\|S_{s}\right\|_{2,1}=\sum_{i=1}^{M}\left\|s^{i}\right\|_{2}$ is the $l_{2,1}$-norm of $S_{s}$ (recall that $s^{i}$ is the $\mathrm{i}$-th row of $S_{s}$ ), and $\lambda$ is a regularization parameter.

Greedy Block Coordinate Descent(GBCD) Algorithm. The objective function in (6) can be written as

$$
F\left(S_{s}\right)=G\left(S_{s}\right)+H\left(S_{s}\right)
$$

Where $G\left(S_{s}\right)=\frac{1}{2}\left\|Y_{s}-\Phi S_{s}\right\|_{F}^{2}=\frac{1}{2}\left\|\operatorname{vec}\left(Y_{s}\right)-I_{R} \otimes \operatorname{Bvec}\left(S_{s}\right)\right\|_{2}^{2}$, and $H\left(S_{s}\right)=\lambda\left\|S_{s}\right\|_{2,1}=\lambda \sum_{i=1}^{M}\left\|s^{i}\right\|_{2}$.

We first build the approximation of $F\left(S_{s}\right)$, that is

$$
\begin{aligned}
F_{\alpha}\left(S_{s}\right) & =G\left(S_{s}(k)\right)+\operatorname{vec}\left(\nabla G\left(S_{s}(k)\right)\right)^{H} \operatorname{vec}\left(S_{s}-S_{s}(k)\right)+\frac{1}{2 \beta}\left\|S_{s}-S_{s}(k)\right\|_{F}^{2}+H\left(S_{s}\right) \\
& =\sum_{i=1}^{M}\left\{\frac{1}{2 \beta}\left\|s^{i}-p^{i}(k)\right\|_{2}^{2}+\lambda\left\|s^{i}\right\|_{2}\right\}+c(k)
\end{aligned}
$$

Here, $\quad P(k)=S_{s}(k)-\beta \nabla G\left(S_{s}(k)\right)=S_{s}(k)-\beta \Phi^{H}\left(\Phi S_{s}(k)-Y_{s}\right) ; s^{i}$ and $p^{i}(k)$ are the i-th rows of $S_{s}$ and $P(k)$, respectively; $c(k)=G\left(S_{s}(k)\right)-\frac{\beta}{2}\left\|\nabla G\left(S_{s}(k)\right)\right\|_{F}^{2} ; \beta$ is chosen to be $\beta=\frac{1}{\left\|\Phi_{i}\right\|_{2}^{2}}$ where $\Phi_{i}$ is any i-th column of $\Phi$.

We can update the $S_{s}(k+1)$ by a soft-thresholding operator

$$
s^{i}(k+1)=\frac{p^{i}(k)}{\left\|p^{i}(k)\right\|_{2}} \max \left(0,\left\|p^{i}(k)\right\|_{2}-\lambda \beta\right)
$$

In order to maintain sparisity during the major iterations, we propose a greedy block selection rule which leads to a greedy block coordinate descent (GBCD) algorithm. Instead if sweeping through all the blocks in parallel, we only update the block that yields the greatest descent distance, namely

$$
i_{0}=\arg \max \left\|s^{i}(k+1)-s^{i}(k)\right\|_{2}, i=1, \ldots, M
$$

Here $\Delta d_{i}=\left\|s^{i}(k+1)-s^{i}(k)\right\|_{2}$ is the descent distance for the i-th block. The greedy block selection rule gives preference to sparsity and has faster convergence. Moreover, it guarantees global convergence.

Based on the MUSIC prior, we choose the weight parameters $\left\{w_{i}\right\}_{i=1}^{M}$ as

$$
w_{i}=\frac{1}{\left\|U_{n}^{H} b_{i}\right\|_{2}}, i=1, \ldots, M
$$

$U_{n}$ is noise subspace of the covariance matrix for received signal. Exploiting the orthogonality between the noise subspace and the array steering vectors, we can improve the accuracy of the GBCD algorithm without changing its global convergence.

\section{SIMULATION}

In this section, we present simulation results illustrating the effectiveness of 2D-GBCD algorithm and 2D-weighted GBCD algorithm for acoustic vector sensor array. We will compare the DOA estimation performance of the proposed two algorithms with L1-SVD for acoustic vector sensor array, such as resolution analysis and error analysis. A 5-element ULA is used with the inter-element spacing being half of a wavelength. The snapshot is 150 and the $\mathrm{SNR}=0 \mathrm{~dB}$. It is assumed that the sources are corrupter by temporally and spatially uncorrelated white Gaussian 
noise, which is measured with signal-to-noise ratio (SNR). In following experiments, the range of angles for DOA is $\left(0,90^{\circ}\right)$, and the grid is uniform with interval being $1^{\circ}$. The source number is assumed to be $K=2$. The source signals impinge upon the acoustic vector-sensor array with $\left(\Phi_{1}, \varphi_{1}\right)=\left(15^{\circ}, 10^{\circ}\right)$, and $\left(\Phi_{2}, \varphi_{2}\right)=\left(60^{\circ}, 70^{\circ}\right)$, repectively.

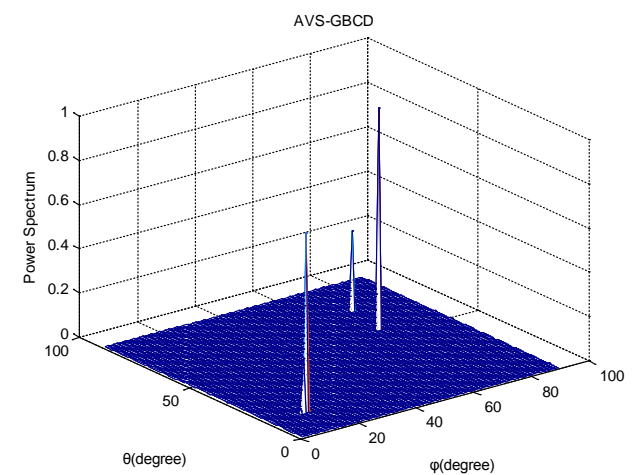

Fig 1. 2-D spatial spectra of GBCD algorithm for AVS array

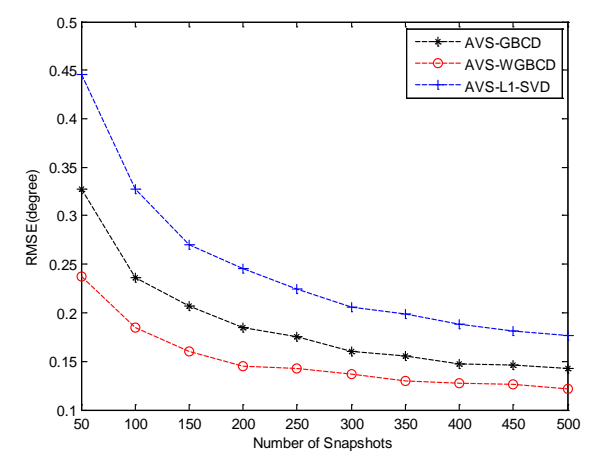

Fig 3. RMSE of 2-D DOA estimation versus number of snapshots

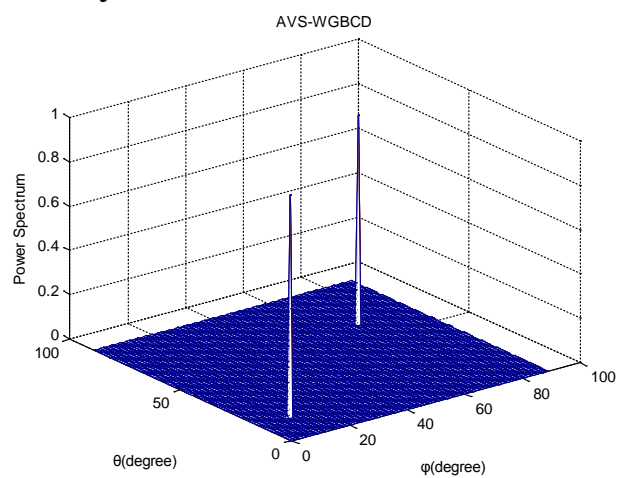

Fig 2. 2-D spatial spectra of weighted GBCD algorithm for AVS array

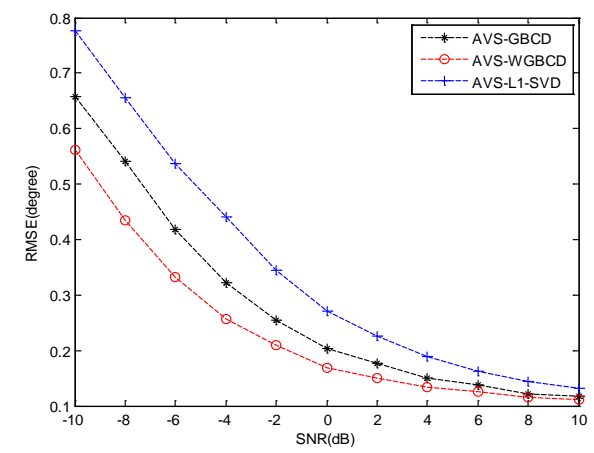

Fig 4. RMSE of 2-D DOA estimation versus SNR

Through the above experiments, it shows that, we can achieve 2-D DOA estimation for acoustic vector sensor array based on spatial sparse sampling. For the figure 1 and figure 2, it is easy to know, the weighted GBCD algorithm for AVS have a better resolution than GBCD algorithm. For the figure 4, we know that W-GBCD algorithm has higher angle resolution than other two algorithms in all SNR and number of snapshots.

\section{Summary}

2D-DOA estimation approach of acoustic vector sensor array based on spatial sparse sampling is proposed. The joint sparse model along with singular value decomposition is utilized to enhance the adaptability to noisy situation and reduce dimension. Finally the high resolution DOA estimation is given by GBCD and weighted GBCD algorithms. Simulation results show that weighted GBCD algorithm has better angle resolution than other approaches in all SNR. The combination of Compressed Sensing and acoustic vector sensor shows great superiority, but how to speed up the recovery algorithms and reduce the sparsity constraints by AVS is worth further researching.

\section{References}

[1] J. Capon, "High resolution frequency-wavenumber spectrum analysis,” Proc. IEEE, vol. 57, no. 8, pp. 1408-1418, Aug. 1969.

[2] R. O. Schmidt, "Multiple emitter location and signal parameter estimation," IEEE Trans. Anten. Propagat., vol. 34, no. 3, pp. 276-280, Mar. 1986. 
[3] R. Roy and T. Kailath, "ESPRIT-Estimation of signal parameters via rotational invariance techniques," IEEE Trans. Acoust., Speech, Signal Process., vol. 37, no. 10, pp. 984-995, Oct. 1989.

[4]A. Nehorai, and E. Paldi, “Acoustic vector-sensor array processing,” IEEE Trans. Sig. Process., vol.42(9),pp.2481-2491, Sep 1994.

[5] M. R. Azimi-Sadjadi, A. Pezeshki and N. Roseveare, "Wideband DOA estimation algorithms for multiple moving sources using unattended acoustic sensors," IEEE Transactions on Aerospace and Electronic Systems, vol. 44(4), pp.1585-1599, 2009.

[6] P. Wang, G. J. Zhang, J. J. Xiong, C. Y. Xue and W. D. Zhang, "Root-MUSIC Algorithm with Real-Valued Eigendecomposition for Acoustic Vector Sensor Array,” Pervasive Computing Signal Processing and Applications (PCSPA), 2010 First International Conference on, pp.652- 656, September 2010.

[7] N. Zou, A. Nehorai, “Circular Acoustic Vector-Sensor Array for Mode Beamforming,” IEEE Transactions on Signal Processing, vol. 57(8), pp.3041-3052, August 2009.

[8] J. He, M. Omair Ahmad, "Joint Space-Time Parameter Estimation for Underwater Communication Channels with Velocity Vector Sensor Arrays," IEEE Transactions on Wireless Communications. vol. 11(11), pp.3869-3877, November 2012.

[9] D. I. Donoho, “Compressed Sensing,” IEEE Transactions on Information Theory, vol. 52(4), pp.1289-1306, 2006.

[10] J. S. Fu, X. K. Li and S. Q. Yu, "Research on parameters estimation of acoustic vector array signals using the compressed sensing theory,” Piezoelectricity, Acoustic Waves and Device Applications (SPAWDA), 2011 Symposium on, pp.138-141, December 2011.

[11] G. L. Liang, W. Ma, Z. Fan and Y. L. Wang, "A high resolution robust localization approach of high speed target based on vector sonar,” Acta Phys. Sin., vol. 62(14), 144302, 2013.

[12] M. Hawkes and A. Nehorai, "Acoustic vector-sensor beamforming and Capon direction estimation,” IEEE Trans. Signal Process., vol. 46, pp. 2291-2304, Sep. 1998.

[13] D. Malioutov, M. Çetin, and A. S. Willsky, "A sparse signal reconstruction perspective for source localization with sensor arrays,” IEEE Trans. Signal Process., vol. 53, no. 8, pp. 3010-3022, Aug. 2005. 